\title{
Multiobjective optimizations of a novel cryocooled dc gun based ultrafast electron diffraction beam line
}

\author{
Colwyn Gulliford, ${ }^{*}$ Adam Bartnik, and Ivan Bazarov \\ CLASSE, Cornell University, 161 Synchrotron Drive, Ithaca, New York 14853-8001, USA
}

(Received 18 April 2016; published 23 September 2016)

\begin{abstract}
We present the results of multiobjective genetic algorithm optimizations of a single-shot ultrafast electron diffraction beam line utilizing a $225 \mathrm{kV}$ dc gun with a novel cryocooled photocathode system and buncher cavity. Optimizations of the transverse projected emittance as a function of bunch charge are presented and discussed in terms of the scaling laws derived in the charge saturation limit. Additionally, optimization of the transverse coherence length as a function of final rms bunch length at the sample location have been performed for three different sample radii: 50, 100, and $200 \mu \mathrm{m}$, for two final bunch charges: $10^{5}$ electrons ( $16 \mathrm{fC}$ ) and $10^{6}$ electrons (160 fC). Example optimal solutions are analyzed, and the effects of disordered induced heating estimated. In particular, a relative coherence length of $L_{c, x} / \sigma_{x}=$ $0.27 \mathrm{~nm} / \mu \mathrm{m}$ was obtained for a final bunch charge of $10^{5}$ electrons and final bunch length of $\sigma_{t} \approx 100 \mathrm{fs}$. For a final charge of $10^{6}$ electrons the cryogun produces $L_{c, x} / \sigma_{x} \approx 0.1 \mathrm{~nm} / \mu \mathrm{m}$ for $\sigma_{t} \approx 100-200 \mathrm{fs}$ and $\sigma_{x} \geq 50 \mu \mathrm{m}$. These results demonstrate the viability of using genetic algorithms in the design and operation of ultrafast electron diffraction beam lines.
\end{abstract}

DOI: 10.1103/PhysRevAccelBeams.19.093402

\section{INTRODUCTION}

Electron diffraction [1] has become a valuable measurement technique with modern ultrafast electron diffraction (UED) experiments [2-9] now capable of atomic level observation of structural dynamics using single-shot tabletop sized setups $[4,7]$. Consequently, the desire for singleshot UED beam lines with increasing coherence continues to push the development of both photocathode and cold atom electron sources [10-16]. Advances in the development of low mean transverse energy (MTE) photocathodes $[17,18]$, as well as both de gun and normal conducting rf (NCRF) gun technology [19,20], promise to provide electron beams with ever increasing brightness (low emittance). Application of this technology in UED beam lines opens up the possibility for atomic resolution of increasingly complicated systems with larger unit cells. The study of biological samples, such as proteins, remains a challenge for single-shot experiments, as they require large transverse coherence $L_{c, x} \gtrsim 1 \mathrm{~nm}$, high bunch charges $q \gtrsim 10^{5}$ electrons, and short pulse lengths $\sigma_{t} \lesssim 100$ fs [8,21].

Designing a photoemission source for these bunch charges and beam sizes implies transporting a strongly space charge dominated beam $[4,8,21,22]$. Building on the successful application of multiobjective genetic algorithm (MOGA) optimized simulations of space charge dominated

\section{*cg248@cornell.edu}

Published by the American Physical Society under the terms of the Creative Commons Attribution 3.0 License. Further distribution of this work must maintain attribution to the author(s) and the published article's title, journal citation, and DOI. beams used in the design and operation of the Cornell photoinjector [23-25], we apply the same techniques to a moderate energy dc gun followed by two solenoids and a NCRF buncher cavity [11-13]. We use the smallest MTEs considered achievable given the excellent vacuum environment provided by this gun technology. In particular, recent work points to the ability to reduce the cathode MTE via cooling of the cathode [18], and data suggests cathode MTEs as low as $5 \mathrm{meV}$ (cathode emittance of $0.1 \mu \mathrm{m} / \mathrm{mm}$ ) may be possible using multiakali antimonide cathodes cooled to $20 \mathrm{~K}$.

This work is structured as follows: first, we briefly review the definition of coherence and the expected scaling with critical initial laser and beam parameters. Next, a detailed description of the beam line setup, and the parameters for optimization is given. The results of an initial round of optimizations of the emittance vs bunch charge, as well as detailed optimizations of the coherence length vs final bunch length at several final beam sizes $\left(\sigma_{x} \approx 25,50,100 \mu \mathrm{m}\right)$ and bunch charges $\left(10^{5}\right.$ and $10^{6}$ electrons) follow. From the optimal fronts, examples for $\sigma_{x} \approx 50 \mu \mathrm{m}$ are simulated for both final charges, and the dynamics in each case discussed.

\section{A. Coherence length from photocathode sources}

The transverse coherence length is defined as $L_{c, x} \approx$ $\hbar / \sigma_{p_{x}}=\chi_{e} / \sigma_{\gamma \beta_{x}} \quad[4,7,8,10-16] \quad$ where $\chi_{e} \equiv \hbar / m_{e} c=$ $3.862 \ldots \times 10^{-4} \mathrm{~nm}$ is the reduced Compton wavelength of the electron. In this and all subsequent expressions, all fields and particle distributions are assumed symmetric about the beam line $(z)$ axis. Rewriting the coherence length in terms of the (normalized) emittance $\epsilon_{n, x}$ gives 


$$
\frac{L_{c, x}}{\chi_{e}}=\frac{1}{\sigma_{\gamma \beta_{x}}}=\frac{\sigma_{x}}{\sqrt{\epsilon_{n, x}^{2}+\left\langle x \cdot \gamma \beta_{x}\right\rangle^{2}}} .
$$

For a beam passing through a waist this expression reduces to $[11,15]$

$$
\left.\frac{L_{c, x}}{\chi_{e}}\right|_{\text {waist }}=\frac{\sigma_{x}}{\epsilon_{n, x}} .
$$

Determining how this quantity scales with the critical initial beam parameters and accelerating field requires relating the initial and final emittances. Factoring out any emittance degrading effects occurring during transport allows one to write the emittance as $\epsilon_{n, x, i}=f_{\epsilon} \cdot \epsilon_{n, x}$ where the factor $f_{\epsilon} \in(0,1]$ determines the degree of emittance preservation. In general, $f_{\epsilon}$ depends strongly on the space charge dynamics along the beam line, which in turn are determined by the initial and final required beam sizes. Additionally, electrons in very cold, dense beams may experience disorder induced heating (DIH) due to strong pointto-point Coulomb interactions after emission from the photocathode [26-28], a process which fundamentally limits the preservation of the cathode emittance. Nonetheless, using this and the expression for the emittance at the cathode $\epsilon_{n, x, i}=\sigma_{x, i} \sigma_{\gamma \beta_{x, i}}$, the coherence length can be written in terms of the magnification $M=\sigma_{x} / \sigma_{x, i}$ from cathode to sample as well as the initial coherence length:

$$
\frac{L_{c, x}}{\chi_{e}}=f_{\epsilon} \frac{\sigma_{x}}{\epsilon_{n, x, i}}=f_{\epsilon} M \cdot \frac{L_{c, x, i}}{\chi_{e}} .
$$

The mean transverse energy (MTE) of the emitted electrons determines the initial coherence length [15,22]:

$$
\frac{L_{c, x, i}}{\chi_{e}}=\frac{1}{\sigma_{\gamma \beta_{x, i}}}=\sqrt{\frac{m_{e} c^{2}}{\mathrm{MTE}}},
$$

while the charge saturation limit, set by the desired extractable charge and cathode field, determines the size of the laser pulse. Following [29,30], we write the aspect ratio of the photoemitted beam as $A=\sigma_{x, i} / \Delta z \approx$ $\sigma_{x, i} / \frac{e E_{0}}{m_{e} c^{2}}\left(c \sigma_{t, i}\right)^{2}$, where $\Delta z \propto \frac{e E_{0}}{m_{e} c^{2}}\left(c \sigma_{t, i}\right)^{2}$ gives the approximate length of the beam at the time of emission in terms of the field at the cathode $E_{0}$ and the laser pulse length $\sigma_{t, i}$. In the charge saturation limit, this yields

$$
\sigma_{x, i} \propto\left\{\begin{array}{c}
\left(q / E_{0}\right)^{1 / 2}, A \gg 1 \text { ("pancake") } \\
\left(q / \sigma_{t, i}\right)^{2 / 3} E_{0}^{-1}, A \leq 1 \text { ("cigar") } .
\end{array}\right.
$$

Thus, the coherence length scales as

$$
\frac{L_{c, x}}{\chi_{e}} \propto f_{\epsilon} \sigma_{x} \sqrt{\frac{m_{e} c^{2}}{\operatorname{MTE}}}\left\{\begin{array}{c}
\left(E_{0} / q\right)^{1 / 2}, A \gg 1 \\
E_{0}\left(\sigma_{t, i} / q\right)^{2 / 3}, A \lesssim 1 .
\end{array}\right.
$$

For beams with a large degree of emittance preservation, $f_{\epsilon} \approx 1$, and the above expression gives the correct scaling
$[29,30]$. Consequently, one goal of this work is to find an optimal beam line setup which comes as close as possible to satisfying this condition.

\section{ONE APPROACH FOR OPTIMAL COHERENCE LENGTH}

Both limits in Eq. (6) make clear that given a desired final spot size $\sigma_{x}$, and charge $q$ at the sample, maximizing coherence length requires larger cathode fields as well as smaller MTEs. In this work, we seek to document the best coherence length achievable from photogun systems delivering the best in MTE technology. To that end, we simulate a dc gun setup, derived from the design of a $250 \mathrm{kV}$ dc gun featuring a $20 \mathrm{~mm}$ cathode-anode gap, and a novel cryocooled photocathode system capable of cooling multialkali cathodes to $20 \mathrm{~K}$ under design and construction at Cornell University. For this work, we specify the same gun geometry and a slightly lower gun voltage of $225 \mathrm{kV}$, in part guided by the empirical data on voltage breakdown and previous voltage demonstration figures for dc guns shown in Fig. 1 [31]. Recently alkali antimonide photocathodes cooled to $90 \mathrm{~K}$ produced MTEs as low as a $15 \mathrm{meV}$ [18]. We anticipate MTEs of a few meV may be achievable in the new cryogun system, and thus, for simplicity, we assume a cathode MTE of $5 \mathrm{meV}$ for all simulations for this beam line.

To model the gun fields, we use an analytic expression for the potential of a plate conductor with a hole in it immersed in a constant background field. For this system, the potential due to anode hole is

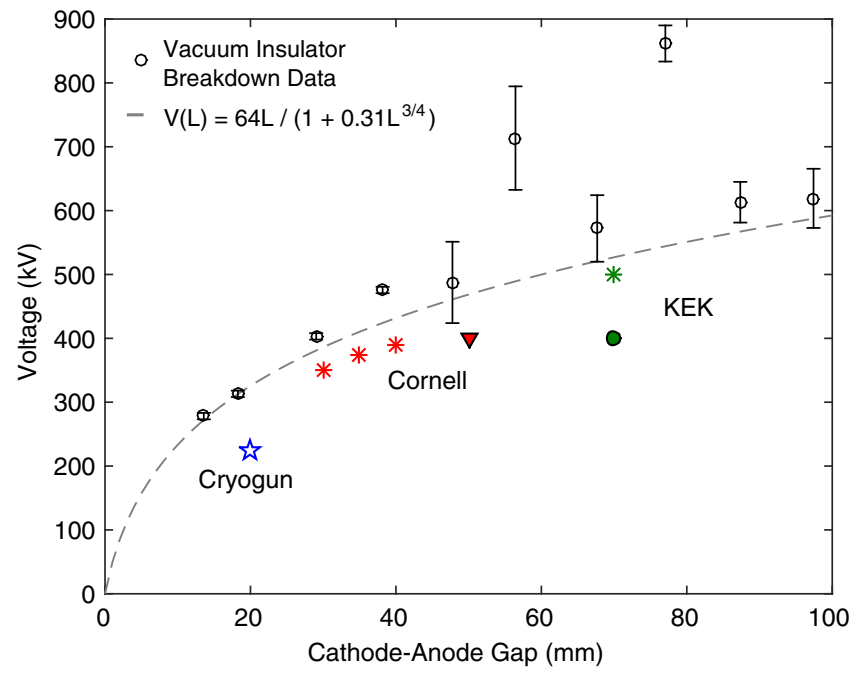

FIG. 1. Voltage performance of high voltage dc systems as a function of the cathode-anode gap: (black) vacuum insulator breakdown data. Additionally, the proposed gap and voltage for the Cornell cryogun studied here, (red) the stable processing voltages and gaps for the Cornell segmented gun with movable anode with $300 \mathrm{pC}$ bunches demonstrated at $400 \mathrm{kV}$ (triangle), processing results of the second generation segmented gun at KEK (green), and voltage for beam tests (circle). 


$$
\begin{aligned}
\Phi(r, z) & =-E_{0}\left(\frac{r_{0}}{\pi}\right)\left[\sqrt{\frac{\rho-\lambda}{2}}-\frac{|z-L|}{r_{0}} \tan ^{-1} \sqrt{\frac{2}{\rho+\lambda}}\right], \\
\rho(r, z) & =\sqrt{\lambda(r, z)^{2}+4(z-L)^{2} / r_{0}^{2}}, \\
\lambda(r, z) & =\frac{1}{r_{0}^{2}}\left[(z-L)^{2}+r^{2}\right]-1 .
\end{aligned}
$$

In this expression, $E_{0}$ is the field at the cathode, $r_{0}$ is the radius of the anode hole, and $L$ is the cathode-anode gap. The full potential is then given by

$$
V(r, z)=\left\{\begin{array}{l}
E_{0} z+\Phi(r, z), \quad \text { if } z<L \\
\Phi(r, z), \quad \text { if } z>L .
\end{array}\right.
$$

This solution becomes exact in the limit $L \rightarrow \infty$ and remains a good approximation provided $r_{0} / L \ll 1$. Here, the radius of the anode hole is $r_{0}=2.5 \mathrm{~mm}$ (compared to $20 \mathrm{~mm}$ for the gap), and the relative voltage error across the cathode is $<1 \%$ :

$$
\left|\frac{\Phi(z=0, r=0)}{\Phi(z=0, r=\infty)}-1\right|<0.01
$$

For this field setup, the $225 \mathrm{kV}$ gun voltage corresponds to a roughly $11 \mathrm{MV} / \mathrm{m}$ accelerating field at the cathode.

Figure 2 shows the overall layout of the cryogun beam line. This setup features a $3 \mathrm{GHz}$ normal conducting buncher cavity for final bunch compression, as well as two solenoid magnets [11-13]. For the buncher fields, we used the same $3 \mathrm{GHz}$ field map as the Eindhoven design [11] (a new $3 \mathrm{GHz}$ design is currently underway at Cornell). The solenoid field maps were created by scaling down the existing Cornell photoinjector fields by a factor of 2. We then fit the analytic form for the on-axis solenoid field from a sheet of current with radius $r_{\text {sol }}$ and length $l_{\text {sol }}$,

$$
B_{z}(z)=B_{0}\left(\frac{\Delta z_{+}}{\sqrt{\Delta z_{+}^{2}+r_{\mathrm{sol}}^{2}}}-\frac{\Delta z_{-}}{\sqrt{\Delta z_{-}^{2}+r_{\mathrm{sol}}^{2}}}\right),
$$

where $\Delta z_{ \pm}=z \pm l_{\text {sol }} / 2$, to the original solenoid field maps. The fields are then modeled with the analytic offaxis expansion of Eq. (10) to third order in the radial offset $r$ in a custom field element for the space charge code General Particle Tracer (GPT) $[32,33]$. We note here that the first-order expansion of the solenoid fields

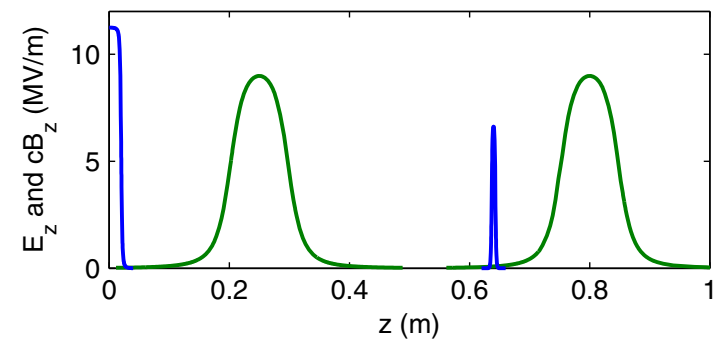

FIG. 2. Example of the on-axis electric field $E_{z}$ (blue) and solenoid field $B_{z}$ profiles for the cryogun setup.
TABLE I. Beam line simulation parameters.

\begin{tabular}{lc}
\hline \hline Parameter & Range \\
\hline Initial charge & {$[0,1000] \mathrm{fC}$} \\
Laser size $\sigma_{t, i}$ & {$[0,20] \mathrm{ps}$} \\
Laser size $\sigma_{x, i}$ & {$[0,1] \mathrm{mm}$} \\
Cathode MTE & $5 \mathrm{meV}$ \\
Peak gun field & $11.1 \mathrm{MV} / \mathrm{m}$ \\
Solenoid 1 peak field & {$[0,0.48] \mathrm{T}$} \\
Solenoid 1 position & {$[0.17,0.67] \mathrm{m}$} \\
Peak buncher field & {$[0.0,15] \mathrm{MV} / \mathrm{m}$} \\
Buncher phase & {$[0,360] \mathrm{deg}$} \\
Buncher position & {$[0.27,1.27] \mathrm{m}$} \\
Solenoid 2 peak field & {$[0.0,0.48] \mathrm{T}$} \\
Solenoid 2 position & {$[0.37,1.87] \mathrm{m}$} \\
Sample position & {$[0.37,3.87] \mathrm{m}$} \\
\hline \hline
\end{tabular}

accurately describes the beam dynamics given the small beam sizes $\left(\sigma_{x} \lesssim 2 \mathrm{~mm}\right)$ determined by the optimizer for both bunch charges. The values for the solenoid radius and length used for this work are 2.94 and $3.32 \mathrm{~cm}$, respectively. Additionally, use of such small MTE values requires estimating the effect of disorder induced heating near the cathode. This issue is discussed later in the results section.

\section{RESULTS}

In order to produce the best coherence length performance from the cryogun UED setup, multiobjective genetic optimizations were performed using GPT and the same optimization software used previously in [23-25]. For these simulations, the optimizer varied the laser rms sizes, beam line element parameters and positions. Additionally, the optimizer was allowed to arbitrarily shape both the transverse and longitudinal laser distributions, based on the same method described in [34]. Table I displays the beam

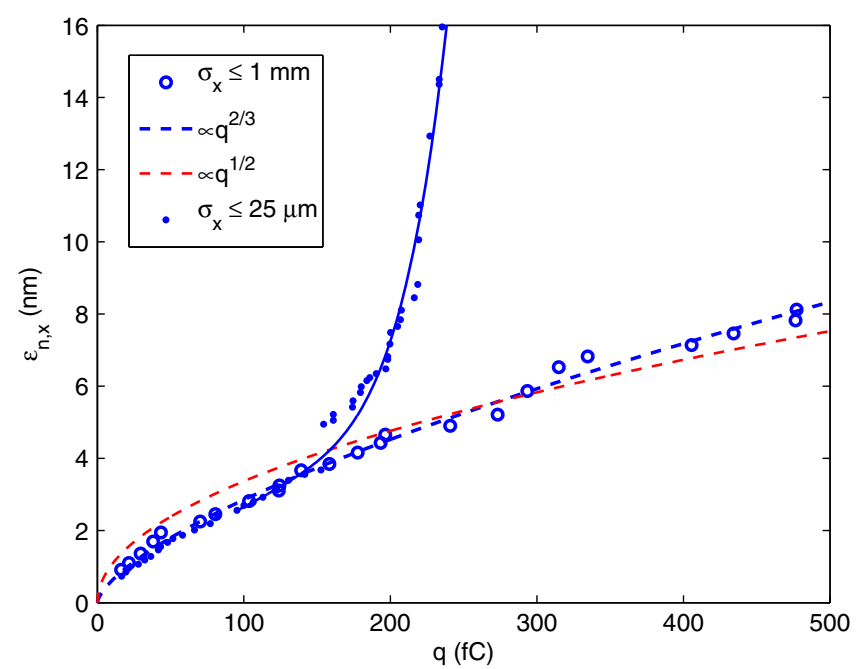

FIG. 3. The optimal emittances for different final transverse spot sizes. Best fits for the two charge scaling regimes are shown in dashed lines. 


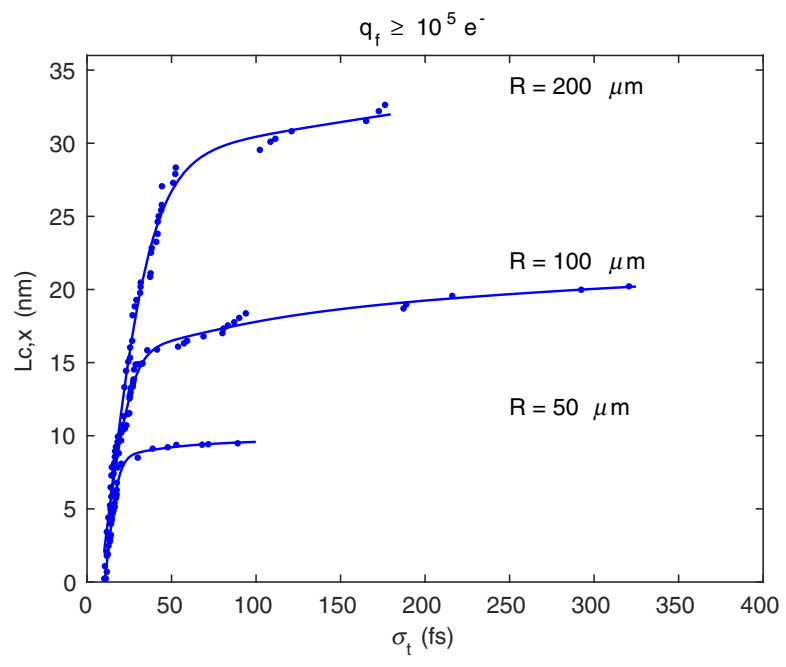

(a) Optimal coherence length vs bunch at the sample.

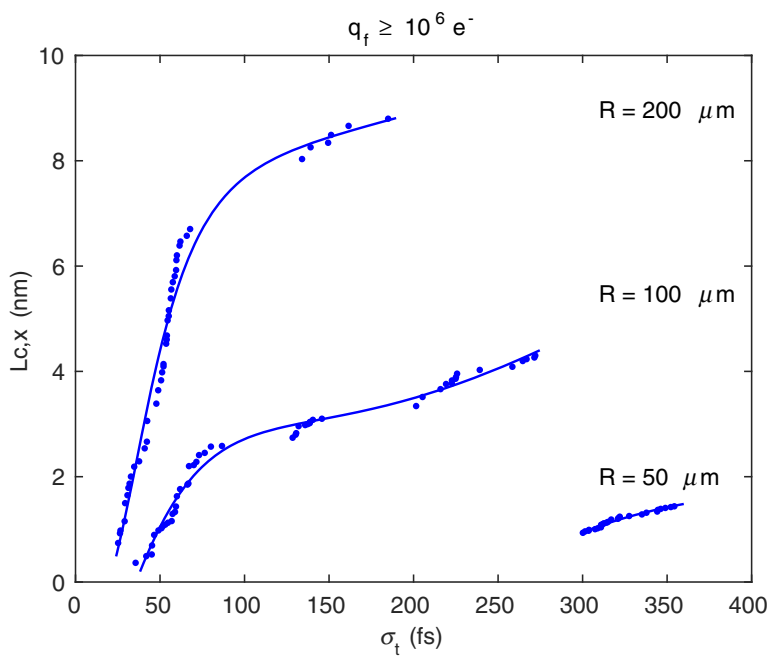

(b) Optimal coherence length vs bunch at the sample.

FIG. 4. Optimal coherence length as a function of bunch length with charge on the sample of (a) $10^{5}$ and (b) $10^{6}$ electrons.

line parameters varied. For all optimizations the population size was set to 50. The number of generations run for each optimization varied between several hundred to a few thousand, depending on if the optimization was starting from noise or being seeded from a previous solution. Convergence was typically judged as follows: once a well-defined front was formed (having run for at least several hundred generations), progression of the front was monitored every 10-20 generations. When no discernible improvement of the front was found, the optimization was stopped.

\section{A. Optimal emittance}

Given a final spot size $\sigma_{x}$ Eqs. (2)-(6) imply the fundamental limit to the coherence is the emittance at the sample. As previously stated, the emittance preservation factor $f_{\epsilon}$ in Eq. (6) determines the degree to which the scaling laws in this expression hold true, and may depend strongly on both the initial and final beam sizes. To determine the effects of constraining the final required rms sizes, we perform an initial round of optimizations for a "large" final beam, $\sigma_{x} \leq 1 \mathrm{~mm}$ and $\sigma_{t} \leq 500 \mathrm{fs}$, and compare that to optimizations with the smallest final spot size considered in this work, $\sigma_{x} \leq 25 \mu \mathrm{m}$. In these optimizations, we require that no particles are lost in beam transport (later we allow for clipping of the beam at the sample), and simultaneously minimize the emittance while maximizing the bunch charge. Figure 3 shows the emittance performance for both spot sizes. In the case of the $25 \mu \mathrm{m}$ spot size, the emittance suffers for charges above roughly $150 \mathrm{fC}$, as the space charge repulsion makes focusing/compressing the bunch down to the desired final beam sizes more difficult.

\section{B. Optimal coherence length}

From the emittance vs charge data in Fig. 3, we selected solutions corresponding to $10^{5}$ and $10^{6}$ electrons at the sample and seeded a new set of optimizations maximizing the coherence length and minimizing the final bunch length at the sample $\sigma_{t}$. The inclusion of a pinhole representing the sample allowed the optimizer to now clip particles at the sample location, subject to the constraint that $q_{f} \geq 10^{5}$ or $q_{f} \geq 10^{6}$ electrons after clipping. For each bunch charge, optimizations were first run with the smallest sample radius $R=50 \mu \mathrm{m}$. These optimizations provided the initial seed for simulations with $R=100 \mu \mathrm{m}$, as the results for the smallest pinhole automatically satisfy all of the constraints for the next larger pinhole. Likewise, the optimization results with $R=100 \mu \mathrm{m}$ provided viable solutions to seed simulations with $R=200 \mu \mathrm{m}$. For all simulations, $6 \mathrm{k}$ macroparticles were used, and the initial charge was allowed to vary up to $1 \mathrm{pC}$, which implies that at least 100 macroparticles must survive the clipping at the sample for the smallest final allowed charge of $q_{f} \geq 10^{5}$ electrons. Upon producing the optimum fronts, additional simulations were run with $30 \mathrm{k}$ macroparticles to check the statistics after clipping, and reproduced the coherence lengths computed with $6 \mathrm{k}$ initial macroparticles to within $20 \%$. In general the emittances computed with $30 \mathrm{k}$ macroparticles were smaller than those at $6 \mathrm{k}$, and correcting for the small shift in the position of the coherence length peak

TABLE II. Relative coherence length values $(\mathrm{nm} / \mu \mathrm{m})$.

\begin{tabular}{lc}
\hline \hline Beam line & $L_{c, x} / \sigma_{x}$ \\
\hline$q_{f} \geq 10^{5} \mathrm{e}^{-}, \sigma_{x} \geq 25 \mu \mathrm{m}, \sigma_{t} \approx 100 \mathrm{fs}$ & 0.27 \\
$q_{f} \geq 10^{6} \mathrm{e}^{-}, \sigma_{x} \geq 50 \mu \mathrm{m}, \sigma_{t} \approx 100 \mathrm{fs}$ & 0.10 \\
\hline \hline
\end{tabular}




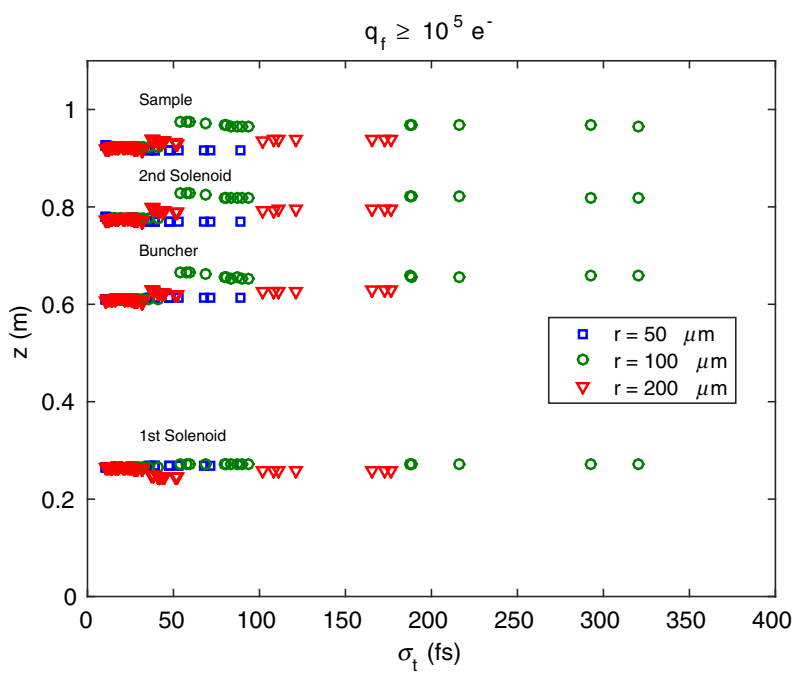

(a) Cryogun beamline element positions.

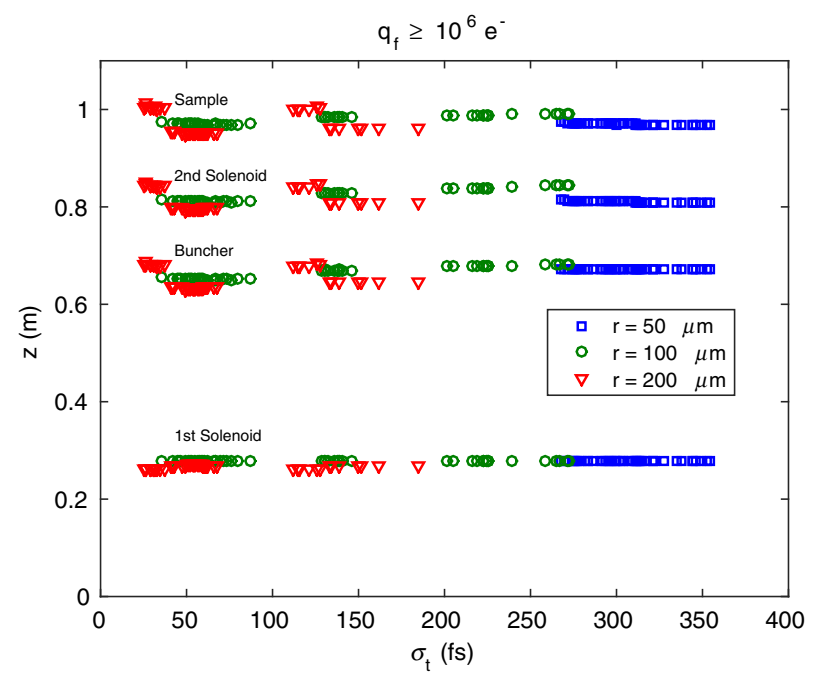

(b) Cryogun beamline element positions.

FIG. 5. Position of the beam line elements for a final charge at the sample of $10^{5}$ electrons (a) and $10^{6}$ electrons (b).

when changing the particle number, the same trend can be seen in the coherence length.

Figure 4 shows the optimal coherence length as a function of final bunch length $\sigma_{t}$ for each bunch charge and sample radius. For $q_{f} \geq 10^{5}$ electrons, the cryogun beam line provides solutions with $\sigma_{t} \lesssim 100$ fs for all three pinhole sizes. Computing the relative coherence length $\left(L_{c, x} / \sigma_{x}\right)$ for a final bunch length of $\sigma_{t} \approx 100 \mathrm{fs}$ using the data from the fits to the optimization results (solid lines) and the fact that the beam size is well approximated as $\sigma_{x} \approx R / 2$, gives $L_{c, x} / \sigma_{x}=0.27 \mathrm{~nm} / \mu \mathrm{m}$. Increasing the required final charge to $q_{f} \geq 10^{6}$ electrons produces more varied coherence performance. For final spot sizes of $\sigma_{x} \geq$ $50 \mu \mathrm{m}$ and final bunch lengths of $\sigma_{t} \approx 200 \mathrm{fs}$, the cryogun beam line produces a relative coherence length of $0.11 \mathrm{~nm} / \mu \mathrm{m}$. For these parameters, estimating the relative coherence length gives $0.1 \mathrm{~nm} / \mu \mathrm{m}$ for a final $\sigma_{t} \leq 100 \mathrm{fs}$. Table II summarizes these values. If the coherence length (considering only the dynamics of the inner portion of the beam that survives clipping) scales as $q_{f}^{-\nu}$, then the ratio of the two required final charges for the curves in Figs. 4(a) and 4(b) implies $\nu=\log _{10}\left[L c, x\left(10^{5} \mathrm{e}^{-}\right) / L c, x\left(10^{6} \mathrm{e}^{-}\right)\right]$. Roughly estimating the coherence length ratios from the asymptotic portions of the solid curves in Figs. 4(a)

TABLE III. Average optimized beam line element positions.

\begin{tabular}{lc}
\hline \hline Element & Position \\
\hline Solenoid 1 & $0.27 \mathrm{~m}$ \\
Buncher cavity & $0.64 \mathrm{~m}$ \\
Solenoid 2 & $0.80 \mathrm{~m}$ \\
Sample pinhole & $0.95 \mathrm{~m}$ \\
\hline \hline
\end{tabular}

and 4(b) gives $\nu=0.76-0.81,0.53-0.6$, and $0.55-0.59$ for the $R=50,100$, and $200 \mu \mathrm{m}$ curves respectively.

In addition to determining the optimal coherence length, the optimizations producing the data in Fig. 4 also provide information about the optimal positioning of the beam line elements in each setup. Figure 5 shows the positions of the beam line elements corresponding to the optimizations shown in Fig. 4. The optimizer eventually settled on fairly fixed element positions for both final charges and all sample radii. Table III displays the

TABLE IV. Relevant example data.

\begin{tabular}{lc}
\hline \hline Parameter & Cryogun \\
\hline (a) $10^{5}$ electrons, $R=100, \mu \mathrm{m} \sigma_{t} \approx 200 \mathrm{fs}$ & \\
Estimated DIH & $0.75 \mathrm{meV}$ \\
Laser $\sigma_{x, y}$ & $5.36 \mu \mathrm{m}$ \\
Laser $\sigma_{t}$ & $8280 \mathrm{fs}$ \\
Aspect ratio A & 0.04 \\
$q_{i}$ & $47.2 \mathrm{fC}$ \\
$q_{f} / q_{i}$ & 0.35 \\
$\epsilon_{n, x}$ & $1.05 \mathrm{~nm}$ \\
$L_{c, x}$ & $18.1 \mathrm{~nm}$ \\
$\chi_{e} \sigma_{x} / \epsilon_{n, x}$ & $18.4 \mathrm{~nm}$ \\
$(\mathrm{~b}) 10^{6}$ electrons, $R=100, \mu \mathrm{m} \sigma_{t} \approx 200 \mathrm{fs}$ & \\
Estimated DIH & $1.6 \mathrm{meV}$ \\
Laser $\sigma_{x, y}$ & $5.83 \mu \mathrm{m}$ \\
Laser $\sigma_{t}$ & $7310 \mathrm{fs}$ \\
Aspect ratio A & 0.06 \\
$q_{i}$ & $239 \mathrm{fC}$ \\
$q_{f} / q_{i}$ & 0.73 \\
$\epsilon_{n, x}$ & $5.27 \mathrm{~nm}$ \\
$L_{c, x}$ & $3.25 \mathrm{~nm}$ \\
$\chi_{e} \sigma_{x} / \epsilon_{n, x}$ & $3.7 \mathrm{~nm}$ \\
\hline \hline
\end{tabular}




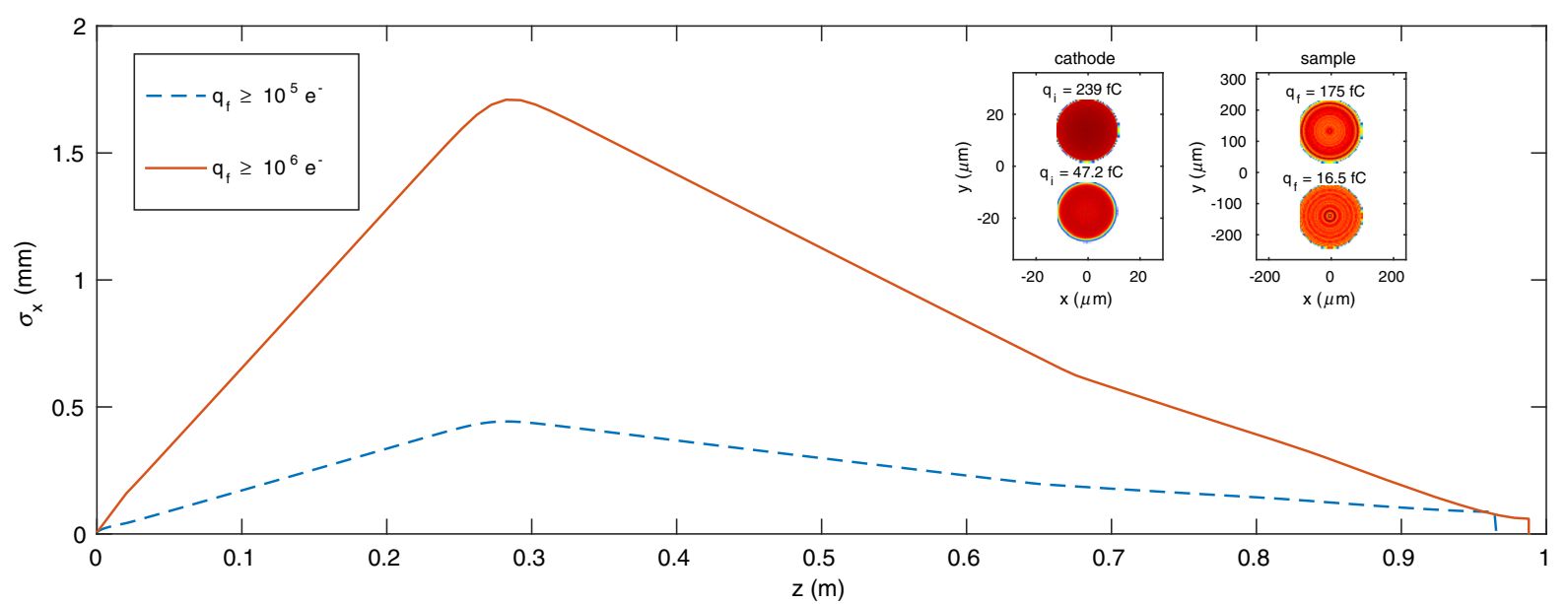

(a)

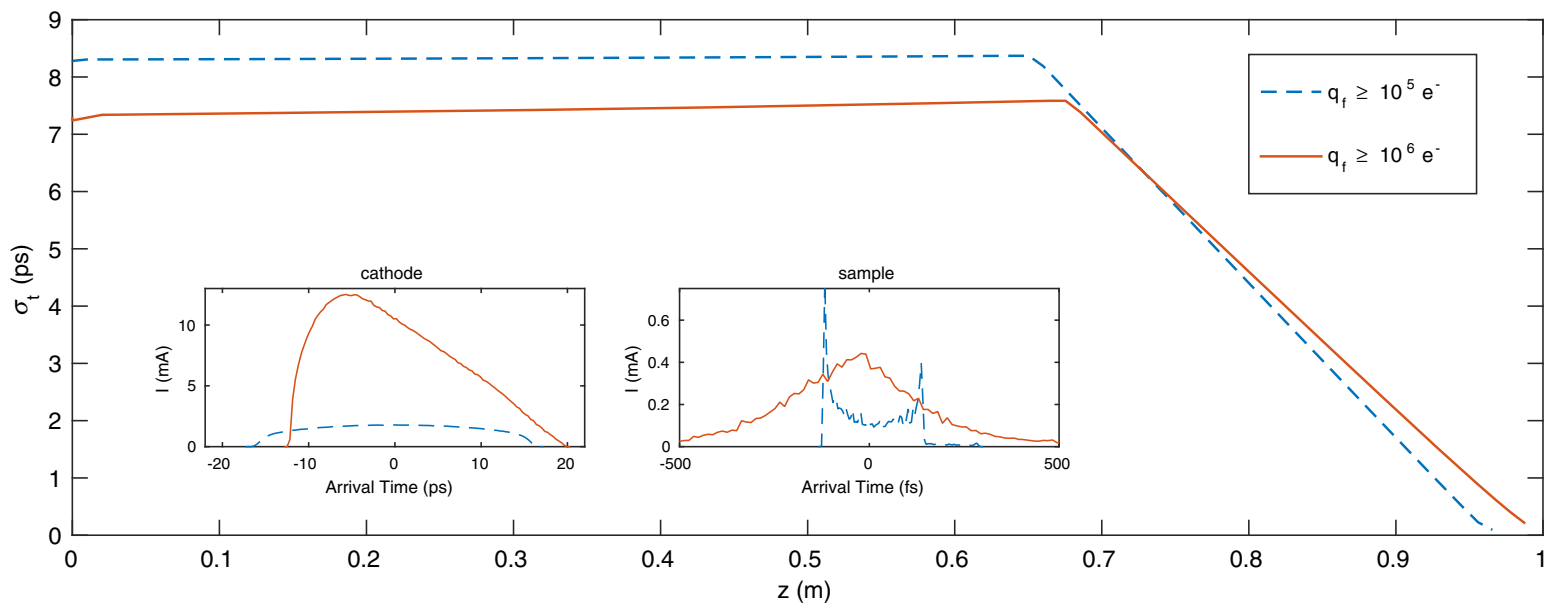

(b)

FIG. 6. Transverse (a) and longitudinal (b) rms beam size along the cyrogun beam line for final charges of $10^{5}$ (dashed line) and $10^{6}$ electrons (solid line). Insets show the transverse (a) and longitudinal (b) laser distributions at the cathode and electron beam distributions at the sample location for both final charges.

element positions averaged over all six optimizations shown in Fig. 4.

\section{Example simulations}

In order to get a better feel of the beam dynamics determined by the coherence length optimizations, we ran several example solutions from the coherence vs final bunch length fronts shown in Fig. 4. From these, we present two examples, one for each of the final charges. In all cases shown, the final sample radius was $R=100 \mu \mathrm{m}$. The final rms bunch lengths were set to $\sigma_{t} \approx 100$ and $200 \mathrm{fs}$ for the lower/higher final charge, respectively. Table IV displays the resulting relevant beam parameters.

Figure 6(a) shows the transverse rms beam size along the cryogun beam line, as well as the initial transverse laser profile and the final electron beam transverse distribution at the sample for both bunch charges. The optimizer chose a roughly flattop transverse laser profile with $\sigma_{x} \approx 5 \mu \mathrm{m}$ for both final charges. The clipping at the sample produces a roughly flattop transverse electron beam distribution, validating the approximation $\sigma_{x} \approx R / 2$ used to compute the relative coherence lengths in Table II. The optimizer chose a smaller transmission $T=q_{f} / q_{i}$ for the smaller final charge $q_{f} \geq 10^{5}$ electrons, with $T=35 \%$ transmission. At $q_{f} \geq 10^{6}$ electrons, the optimizer clipped fewer particles, resulting in a transmission of $T=73 \%$.

Figure 6(b) shows the rms bunch length, and the initial temporal current profile produced by the laser, and the electron beam current profile at the sample. The use of the buncher cavity allows for a fairly constant bunch length along the beam line up to the cavity, where the buncher applies an energy chirp which results in the bunch being compressed by the time it reaches the screen. The optimizer chose a roughly flattop longitudinal initial laser distribution for the lower charge, and a sloped distribution at the higher charge.

From the transverse and longitudinal rms data, the initial electron beam volume and aspect ratio follow, which allows 


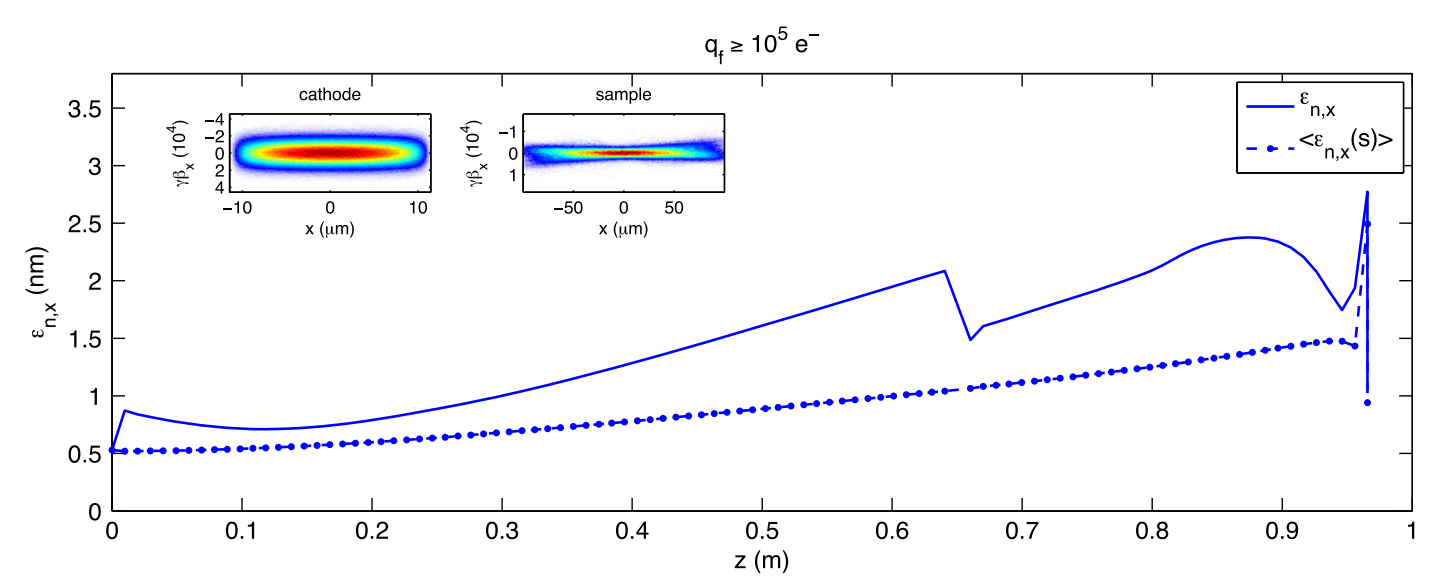

(a)

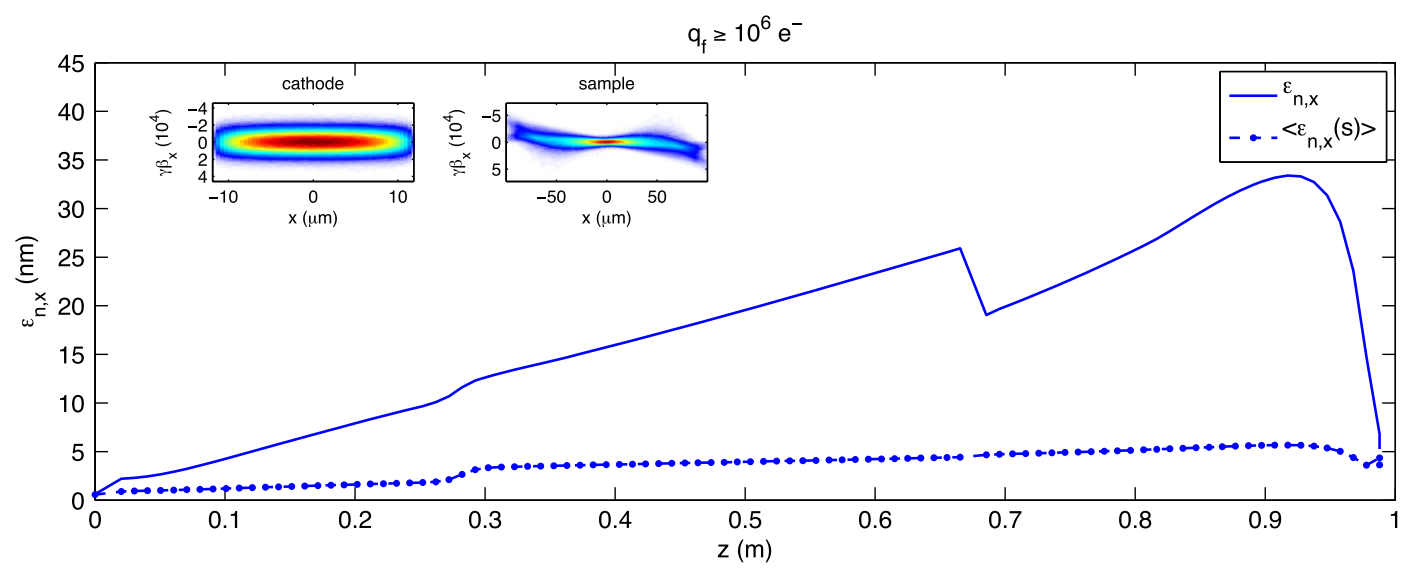

(b)

FIG. 7. Transverse rms projected and average slice emittance along the cyrogun beam line for a final charge of $10^{5}$ electrons (a) and $10^{6}$ (b) electrons. Insets show the transverse phase space distributions at the cathode and sample locations.

for the estimation of the disordered induced heating near the cathode surface, as well as which scaling law regime from Eq. (6) should apply to the dynamics. In both cases, we assume a uniform beam with equivalent rms sizes. From this, the volume follows:

$$
\begin{aligned}
V & =\pi R^{2} L \approx \pi\left(2 \sigma_{x}\right)^{2} \cdot \frac{1}{2} \frac{e E_{0}}{m}\left(\sqrt{12} \sigma_{t}\right)^{2} \\
& \approx \frac{24 \pi E_{0}}{m c^{2}[\mathrm{eV}]} \sigma_{x}^{2}\left(c \sigma_{t}\right)^{2} .
\end{aligned}
$$

Using this to compute the electron number density for each of the example cases yields $4 \times 10^{17}\left(4 \times 10^{18}\right) \mathrm{m}^{-3}$ for the final charges at the sample of $10^{5}\left(10^{6}\right)$ electrons respectively. To estimate the DIH, we note that significant heating occurs when the average potential energy between nearest neighbor electrons after photoemission is greater than or on the order of the electron's thermal energy $k T \lesssim e^{2} / 4 \pi \epsilon_{0} a$, where $a=\left(3 / 4 \pi n_{0}\right)^{1 / 3}$ is the Wigner-Seitz radius [27,28]. For a beam with zero initial temperature, the effective heating then takes the form $\Delta k T=\alpha n_{0}^{1 / 3}$. The constant of proportionality for a static bunch has been computed by
Maxson, resulting in $\Delta k T[\mathrm{eV}]=1.04 \times 10^{-9}\left(n_{0}\left[\mathrm{~m}^{-3}\right]\right)^{1 / 3}$ [27]. Using this expression for the example bunches yields an estimated DIH effect of 0.75 and $1.6 \mathrm{meV}$ for the lower/higher final sample charge, or roughly $15 \%$ and $32 \%$ of the original $5 \mathrm{meV}$ cathode MTE. Computing the initial electron beam aspect ratio yields $A=0.04(0.06)$ with a final charge of $10^{5}\left(10^{6}\right)$ electrons, respectively. As anticipated from the emittance optimizations, the cryogun produces best performance when operating in the long initial electron beam limit.

As the emittance performance largely determines the optimal coherence length, we also plot both the transverse projected and average slice emittance along each beam line for each final charge. For the slice emittance calculation, each simulation was run with $30 \mathrm{k}$ macroparticles, and binned using 20 longitudinal slices along the bunch. The emittance in slice, $\epsilon_{n, x}(s)$, was computed and then the average over the slices taken to get a single number representative of the slice data. Figure 7 (a) shows the emittances computed using the lower final charge for the cryogun. Shown in the insets are the initial and final horizontal phase spaces in both cases. The space charge 


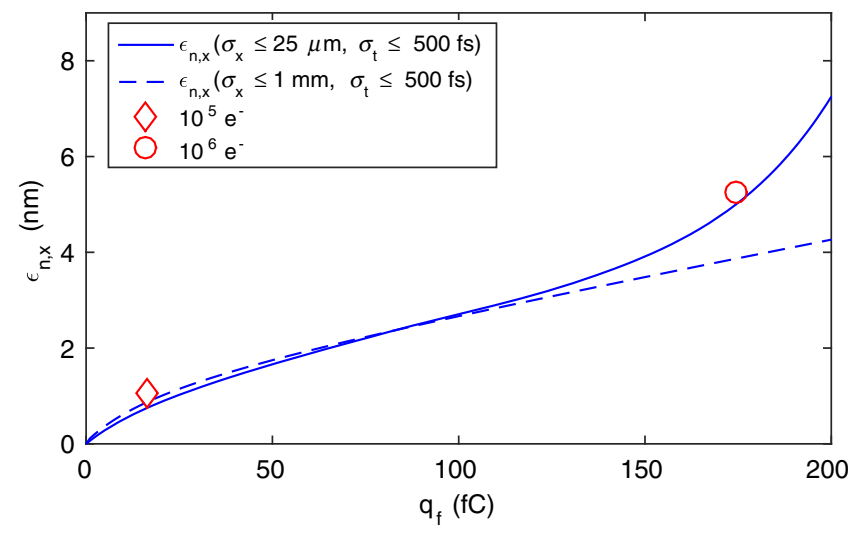

FIG. 8. Comparison of the optimized emittance with $\sigma_{t} \leq$ $500 \mathrm{fs}$ and $\sigma_{x}<25 \mu \mathrm{m}$ (solid line) and $\sigma_{x}<1 \mathrm{~mm}$ (dashed line) and the emittance resulting from the coherence length optimizations (red) with $\sigma_{t} \leq 100(200)$ fs at $10^{5}\left(10^{6}\right)$ electrons respectively.

induced rotation of the slices grows the projected emittance along the beam line up to the point of the last solenoid before the sample, which is used to send the beam through a waist, aligning the slices in the process. We point out that the emittance drops following the eventual slice realignment due to the second solenoid. However, the emittance blows up again as the beam is compressed longitudinally before being clipped at the sample, after which the emittance is on the order of $1 \mathrm{~nm}$. Figure 7(b) shows the corresponding data for the final charge of $q_{f} \geq 10^{6}$ electrons. The dynamics are similar to the lower charge, though emittance is significantly larger along the beam line. The curvature of the final phase in this case indicates the bunch has experienced nonlinear fields along transport, which is verified by the increase of the average slice emittance along the beam line. Table IV collects all of the relevant emittance data from these simulations, including the estimate of the coherence using Eq. (2).

Finally, to put the results of the above examples into perspective, we compare the emittances (shown in red) in these results to the optimized emittances for longer bunch lengths (shown in blue). Figure 8 shows the comparison. As anticipated, the emittances in both cases agree nicely, suggesting the optimizer compensates the requirement of additional bunch compression by clipping out particles (hence the smaller particle transmission at the sample). These results show that, even when including particle clipping at the sample, the optimized emittances for given final rms transverse and longitudinal beam sizes correctly estimate the coherence length performance.

\section{CONCLUSIONS}

In this work, we have generated an optimized UED layout through the use of MOGA optimization. The beam line is comprised of a $225 \mathrm{kV}$ dc gun featuring a cryocooled photocathode, a separate bunching cavity, and two solenoid magnets. The design of the $\mathrm{dc}$ gun, in particular the operating voltage $(225 \mathrm{kV})$ and cathode gap $(20 \mathrm{~mm})$, represents a realistically feasible solution based on empirical high voltage break down limits. Additionally, the use of a $5 \mathrm{meV}$ cathode MTE reflects the ultimate performance anticipated for cryocooled multiakali antimonide cathodes. All simulations performed made use of realistic field maps and field models which include nonlinear field effects.

Optimizations of the emittance as a function of bunch charge demonstrate a $q^{2 / 3}$ dependence, as anticipated from the basic scaling laws for a long initial beam at the cathode shown here and derived in the literature. Direct optimization of the coherence length as a function of final bunch length produced a roughly meter long beam line (cathode to sample) with physically realizable element positions. Example solutions from the optimum coherence length fronts demonstrate reasonable beam dynamics for $10^{5}$ and $10^{6}$ electrons. The effect of disorder induced heating, a process which may limit the brightness of photogun sources, has been estimated and shown to be on the order of $1.6 \mathrm{meV}$ or less for the examples discussed. The coherence and bunch length data produced here using MOGA optimization demonstrates the viability of the presented beam line as a tabletop, single-shot UED setup, with bunch parameters suitable for use with protein samples. Work is currently underway at Cornell to realize this design.

\section{ACKNOWLEDGMENTS}

This grant was supported by the NSF, Award No. PHY 1416318.

[1] C. Davisson and L. H. Germer, Diffraction of electrons by a crystal of nickel, Phys. Rev. 30, 705 (1927).

[2] J. C. Williamson, J. Cao, H. Ihee, H. Frey, and A. H. Zewail, Clocking transient chemical changes by ultrafast electron diffraction, Nature (London) 386, 159 (1997).

[3] H. Ihee, V. A. Lobastov, U. M. Gomez, B. M. Goodson, R. Srinivasan, C.-Y. Ruan, and A. H. Zewail, Direct imaging of transient molecular structures with ultrafast diffraction, Science 291, 458 (2001).

[4] B. J. Siwick, J. R. Dwyer, R. E. Jordan, and R. J. D. Miller, Ultrafast electron optics: Propagation dynamics of femtosecond electron packets, J. Appl. Phys. 92, 1643 (2002).

[5] B. J. Siwick, J. R. Dwyer, R. E. Jordan, and R. J. D. Miller, An atomic-level view of melting using femtosecond electron diffraction, Science 302, 1382 (2003).

[6] M. Harb, R. Ernstorfer, C. T. Hebeisen, G. Sciaini, W. Peng, T. Dartigalongue, M. A. Eriksson, M. G. Lagally, S. G. Kruglik, and R. J. D. Miller, Electronically Driven Structure Changes of $\mathrm{Si}$ Captured by Femtosecond Electron Diffraction, Phys. Rev. Lett. 100, 155504 (2008). 
[7] G. Sciaini, M. Harb, S. G. Kruglik, T. Payer, C. T. Hebeisen, F.-J. M. z. Heringdorf, M. Yamaguchi, M. H. -v. Hoegen, R. Ernstorfer, and R. J. D. Miller, Electronic acceleration of atomic motions and disordering in bismuth, Nature (London) 458, 56 (2009).

[8] G. Sciaini and R. J.D. Miller, Femtosecond electron diffraction: heralding the era of atomically resolved dynamics, Rep. Prog. Phys. 74, 096101 (2011).

[9] S. Lahme, C. Kealhofer, F. Krausz, and P. Baum, Femtosecond single-electron diffraction, Struct. Dyn. 1, 034303 (2014).

[10] B. Siwick, J. Dwyer, R. Jordan, and R. Miller, Femtosecond electron diffraction studies of strongly driven structural phase transitions, Chem. Phys. 299, 285 (2004).

[11] T. van Oudheusden, E. F. de Jong, S. B. van der Geer, W. P. E. M. O. 't Root, O. J. Luiten, and B. J. Siwick, Electron source concept for single-shot sub-100 fs electron diffraction in the $100 \mathrm{keV}$ range, J. Appl. Phys. 102, 093501 (2007).

[12] T. van Oudheusden, P. L. E. M. Pasmans, S. B. van der Geer, M. J. de Loos, M. J. van der Wiel, and O. J. Luiten, Compression of Subrelativistic Space-Charge-Dominated Electron Bunches for Single-Shot Femtosecond Electron Diffraction, Phys. Rev. Lett. 105, 264801 (2010).

[13] R. P. Chatelain, V. R. Morrison, C. Godbout, and B. J. Siwick, Ultrafast electron diffraction with radio-frequency compressed electron pulses, Appl. Phys. Lett. 101, 081901 (2012).

[14] M. Gao, C. Lu, H. Jean-Ruel, L. C. Liu, A. Marx, K. Onda, S.-y. Koshihara, Y. Nakano, X. Shao, T. Hiramatsu, G. Saito, H. Yamochi, R. R. Cooney, G. Moriena, G. Sciaini, and R. J. D. Miller, Mapping molecular motions leading to charge delocalization with ultrabright electrons, Nature (London) 496, 343 (2013).

[15] W. J. Engelen, M. A. van der Heijden, D. J. Bakker, E. J. D. Vredenbregt, and O. J. Luiten, High-coherence electron bunches produced by femtosecond photoionization, Nat. Commun. 4, 1693 (2013).

[16] A. J. McCulloch, D. V. Sheludko, M. Junker, and R. E. Scholten, High-coherence picosecond electron bunches from cold atoms, Nat. Commun. 4, 1692 (2013).

[17] J. Maxson, L. Cultrera, C. Gulliford, and I. Bazarov, Measurement of the tradeoff between intrinsic emittance and quantum efficiency from a NaKSb photocathode near threshold, Appl. Phys. Lett. 106, 234102 (2015).

[18] L. Cultrera, S. Karkare, H. Lee, X. Liu, and I. Bazarov, Cold electron beams from cryo-cooled, alkali antimonide photocathodes, http://arxiv.org/abs/1504.05920.

[19] J. B. Hastings, F. M. Rudakov, D. H. Dowell, J. F. Schmerge, J. D. Cardoza, J. M. Castro, S. M. Gierman, H. Loos, and P. M. Weber, Ultrafast time-resolved electron diffraction with megavolt electron beams, Appl. Phys. Lett. 89, 184109 (2006).

[20] P. Musumeci, J. T. Moody, R. J. England, J. B. Rosenzweig, and T. Tran, Experimental Generation and
Characterization of Uniformly Filled Ellipsoidal ElectronBeam Distributions, Phys. Rev. Lett. 100, 244801 (2008).

[21] R. J. D. Miller, Mapping atomic motions with ultrabright electrons: the chemists' gedanken experiment enters the lab frame., Annu. Rev. Phys. Chem. 65, 583 (2014).

[22] A. M. Michalik and J. E. Sipe, Analytic model of electron pulse propagation in ultrafast electron diffraction experiments, J. Appl. Phys. 99, 054908 (2006).

[23] C. Gulliford, A. Bartnik, I. Bazarov, L. Cultrera, J. Dobbins, B. Dunham, F. Gonzalez, S. Karkare, H. Lee, H. Li, Y. Li, X. Liu, J. Maxson, C. Nguyen, K. Smolenski, and Z. Zhao, Demonstration of low emittance in the Cornell energy recovery linac injector prototype, Phys. Rev. ST Accel. Beams 16, 073401 (2013).

[24] C. Gulliford, A. Bartnik, I. Bazarov, B. Dunham, and L. Cultrera, Demonstration of cathode emittance dominated high bunch charge beams in a DC gun-based photoinjector, Appl. Phys. Lett. 106, 094101 (2015).

[25] A. Bartnik, C. Gulliford, I. Bazarov, L. Cultera, and B. Dunham, Operational experience with nanocoulomb bunch charges in the Cornell photoinjector, Phys. Rev. ST Accel. Beams 18, 083401 (2015).

[26] S. B. van der Geer, M. P. Reijnders, M. J. de Loos, E. J. D. Vredenbregt, P. H. A. Mutsaers, and O. J. Luiten, Simulated performance of an ultracold ion source, J. Appl. Phys. 102, 094312 (2007).

[27] J. M. Maxson, I. V. Bazarov, W. Wan, H. A. Padmore, and C. E. Coleman-Smith, Fundamental photoemission brightness limit from disorder induced heating, New J. Phys. 15, 103024 (2013).

[28] D. Murphy, R. E. Scholten, and B. M. Sparkes, Increasing the Brightness of Cold Ion Beams by Suppressing Disorder-Induced Heating with Rydberg Blockade, Phys. Rev. Lett. 115, 214802 (2015).

[29] I. V. Bazarov, B. M. Dunham, and C. K. Sinclair, Maximum Achievable Beam Brightness from Photoinjector, Phys. Rev. Lett. 102, 104801 (2009).

[30] D. Filippetto, P. Musumeci, M. Zolotorev, and G. Stupakov, Maximum current density and beam brightness achievable by laser-driven electron sources, Phys. Rev. ST Accel. Beams 17, 024201 (2014).

[31] J. Maxson, I. Bazarov, B. Dunham, J. Dobbins, X. Liu, and K. Smolenski, Design, conditioning, and performance of a high voltage, high brightness dc photoelectron gun with variable gap, Rev. Sci. Instrum. 85, 093306 (2014).

[32] Pulsar website for GPT, http://www.pulsar.nl/gpt/.

[33] S. van der Geer, M.d. L. O. J. Luiten, G. Pöplau, and U. van Rienen, 3D space-charge model for GPT simulations of high brightness electron bunches (2005), p. 101.

[34] I. V. Bazarov, A. Kim, M. N. Lakshmanan, and J. M. Maxson, Comparison of dc and superconducting rf photoemission guns for high brightness high average current beam production, Phys. Rev. ST Accel. Beams 14, 072001 (2011). 